Department of Mathematics and Physics

OCU-PHYS-334

Osaka City University

AP-GR-79

\title{
Visible borders of spacetime generated by high-energy collisions
}

\author{
Ken-ichi Nakao ${ }^{1 *}$, Tomohiro Harada ${ }^{2 \dagger}$ and Umpei Miyamoto ${ }^{2 \ddagger}$ \\ ${ }^{1}$ Department of Physics, Graduate School of Science, \\ Osaka City University, Osaka 558-8585, Japan \\ ${ }^{2}$ Department of Physics, Rikkyo University, \\ Toshima, Tokyo 171-8501, Japan.
}

(Dated: April 23, 2018)

\begin{abstract}
Several years ago, two of the present authors proposed the concept of the border of spacetime as a generalization of spacetime singularities. Visible borders of spacetime, which replace naked singularities of classical theory, are not only necessary for the mathematical completeness of general relativity but they also provide a window into new physics of strongly curved spacetime, which is observable in principle. By employing simple geometrical and dimensional arguments, we show that not only black holes but also visible borders of spacetime will be generated at, for example, the CERN Large Hadron Collider, provided that the energy scale of quantum gravity is near $1 \mathrm{TeV}$ in the framework of the large extra-dimension scenario.
\end{abstract}

PACS numbers: 04.20.Dw, 04.20.Cv, 11.10.Kk, 04.62.+v

\footnotetext{
* Electronic address: knakao@sci.osaka-cu.ac.jp

$\dagger$ Electronic address: harada@rikkyo.ac.jp

$\ddagger$ Electronic address: umpei@rikkyo.ac.jp
} 
In the framework of the large extra-dimension scenario, the fundamental Planck scale $E_{P}$ can be lowered to a terra-electron-volt $(\mathrm{TeV})$ scale to resolve the hierarchy problem between the four-dimensional Planck scale $\left(10^{19} \mathrm{GeV}\right)$ and the electroweak scale $(100 \mathrm{GeV})$ [2, 3]. One of the exciting predictions of this scenario is that substantial black holes may be generated at the CERN Large Hadron Collider (LHC) and identified through Hawking radiation 46]. Black holes are one key to solving problems related to the quantum nature of gravity. Hence, the observation of black holes at LHC would represent the first experimental study of quantum gravity.

However, here we consider whether quantum effects of gravity can be observed only through black holes. Within the framework of classical general relativity, singularity theorems predict that development from generic initial data results in singular spacetime [1]. Although these theorems do not specify the nature of spacetime singularities (beside their geodesic incompleteness), these singularities will be accompanied by divergences in the energy density, stress and spacetime curvature (i.e., tidal forces), etc. The big bang is the most well-known example of spacetime singularities. Since all the known laws of physics (including general relativity itself) will lose their validity at spacetime singularities, it is not possible to predict what happens there without knowledge of new physics such as a quantum theory of gravity. A spacetime singularity that can causally affect regular regions is termed a naked singularity. If naked singularities exist, it should, in principle, be possible to observe something from these singularities. The big bang can be regarded as a naked singularity and hence if we obtain any direct signals from it, it will be possible to learn something about the quantum theory of gravity. If naked singularities besides the big bang exist in our universe, they might also be arenas of quantum gravity.

Penrose proposed a conjecture, known as the cosmic censorship hypothesis, regarding the visibility of spacetime singularities [7]. There are two versions of this hypothesis. For spacetimes that contain physically reasonable matter fields and that are developed from generic nonsingular initial data, the weak version of the hypothesis claims that no spacetime singularity is visible from infinity, whereas the strong version claims that the spacetime is globally hyperbolic, or roughly speaking, that there is no unpredictable region in the future of the initial data. A spacetime singularity that conflicts with the weak version is called a globally naked singularity, whereas a naked singularity that does not conflict with the weak version is called a locally naked singularity. This hypothesis plays a crucial role in the proofs 
of theorems for black holes and of singularity theorems. If it is true, it will be impossible to observe spacetime singularities besides the big bang. However, several candidates for counterexamples to the cosmic censorship hypothesis has been theoretically found [8]. Charged black holes and rotating black holes are regarded as serious counterexamples to the strong version of the hypothesis since there are unpredictable regions within charged or rotating black holes, although the existence of such unpredictable regions is not necessarily related to naked singularities [9-12].

It seems likely that spacetime singularities are accompanied by very strong gravity and thus are regarded as fatal disasters. However, this is not always true. Spacetime singularities with strong gravity are surrounded by a domain from which even light rays cannot escape. Such singularities are enclosed by horizons and thus cannot be counterexamples to the weak version of the cosmic censorship hypothesis. In the case of collapse of matter fields due to their self-gravity, which is so strong that it overcomes their own stress, the resultant singularities are not expected to be globally naked. In other words, a naked singularity can be globally naked due to the weakness of its gravity, which is too weak to trap light rays within the vicinity of the singularity. If this is true, the formation of globally naked singularities requires matter that has stress that is too weak to interrupt the collapse caused by the relatively weak self-gravity. Thus, in the cosmic censorship formulated by Wald, physically natural matter fields should not form singularities if there is no gravity [1, 13]. In fact, all known candidates for counterexamples to the weak cosmic censorship hypothesis involve matter fields that do not satisfy Wald's condition or have not been shown to develop from generic nonsingular initial data.

The cosmic censorship hypothesis is formulated within the framework of general relativity. However, since general relativity is a classical theory, it becomes invalid at energy scales higher than the fundamental Planck scale $E_{P}$. Thus, it is more practical to regard a region in which general relativity loses its validity as an effective spacetime singularity. Based on this point of view, Harada and Nakao proposed the concept of the border of spacetime [14]. The domain $\mathcal{A}$ of spacetime is called a border if the following inequality is satisfied:

$$
\inf _{\mathcal{A}} F \geq \alpha E_{P}^{2}
$$

where $\alpha$ is a positive constant of order unity and $F$ is given, for instance, by using the scalar polynomials of the Riemann tensor, or components of the Riemann tensor with respect to a 
parallelly transported orthonormal basis $e_{\mu}^{a}$ as

$$
F:=\max \left(\left|R_{a}^{a}\right|,\left|R^{a b} R_{a b}\right|^{1 / 2},\left|R^{a b c d} R_{a b c d}\right|^{1 / 2},\left|R_{\mu \nu \rho \sigma}\right|\right)
$$

(Hereafter we adopt the natural units $c=\hbar=1$ and the abstract index notation: Latin indices denote the tensor type and Greek indices denote the component with respect to some basis vectors [1].) We denote the union of all borders in spacetime $\mathcal{M}$ by $\mathcal{U}_{\mathrm{B}}$. We call a border $\mathcal{A}$ a visible border if $J^{+}(\mathcal{A}, \mathcal{M}) \cup\left(\mathcal{M}-\mathcal{U}_{\mathrm{B}}\right)$ is not empty, where $J^{+}(\mathcal{A}, \mathcal{M})$ is the causal future of $\mathcal{A}$ in $\mathcal{M}$. We can also naturally introduce a globally visible border as an extension of a globally naked singularity [14].

It is instructive to consider cylindrically symmetric gravitational collapse in fourdimensional spacetime that is asymptotically flat in the spacelike direction orthogonal to the symmetry axis. No horizon forms in this system provided the stress-energy tensor $T_{a b}$ satisfies $T_{a b} k^{a} k^{b} \geq 0$ for any null vector $k^{a}$ [15]. Thus, if a spacetime singularity forms, it is necessarily naked. Several studies of this cylindrical system suggest that it is probable that matter fields with positive tangential stresses that satisfy physically reasonable equations of state do not form a spacetime singularity in gravitational collapse since the tangential stress will finally overcome the cylindrical gravity and, as a result, a bounce will occur [16 19]. On the other hand, if the initial imploding velocity is sufficiently large, the energy density and stress in the vicinity of the symmetry axis can be momentarily very large. In addition, $F$ defined by Eq. (2) can also become very large through the Einstein equations. Thus, in such a situation, the neighborhood of the symmetry axis can be a globally visible border of spacetime, even though the spacetime remains regular.

Giddings and Thomas [4] and Dimopoulos and Landsberg [5] independently pointed out that if the $\mathrm{TeV}$ scale gravity scenario is true, since gravitational attraction in a short range is much stronger than that in a long range, collisions of protons will generate so many black holes at the LHC that the signals of their production should be observable (an excellent review of this is given in Ref. [20]). At the high-energy collider, particles are accelerated and forced to collide with each other. In other words, a very high energy density region will appear due to high initial velocities and, in this sense, the situation is similar to the formation of a cylindrical border mentioned above. Thus, not only black holes but also globally visible borders might form at the LHC. We are investigating whether a region with a very high energy density appears without black hole formation. 
In this paper, we consider a collision of two elementary particles. For simplicity, we assume that both particles are identical and structureless. The extra dimensions are assumed to be compactified into a length scale considerably larger than the fundamental Planck length $E_{P}^{-1}$, whereas the energy of the particles is assumed to be confined within the length scale $E_{P}^{-1}$ in every direction of the extra dimensions; i.e., within a volume $V_{D-4} E_{P}^{-(D-4)}$ in the extra dimensions, where $V_{n}$ is the volume of a unit $n$-ball

$$
V_{n}=\pi^{\frac{n}{2}} / \Gamma\left(\frac{n}{2}+1\right),
$$

where $\Gamma(x)$ is the gamma function. Then, we consider the distribution of two particles in the remaining three-dimensional space.

We denote the center-of-mass (CM) energy of the two particles by $E$, which is much larger than their rest masses, and assume that the three momenta of these particles are parallel to each other. The minimum uncertainty in the position of each particle measured in the longitudinal direction of their three momenta is approximately $E^{-1}$ due to the Lorentz contraction.

In Refs. [4] and [5], it is assumed that these two particles will form a black hole if they pass through a transverse disk of radius $R_{\mathrm{g}}$ at almost the same time, where $R_{\mathrm{g}}$ is the horizon radius of the Schwarzschild-Tangherlini black hole with gravitational mass $E$,

$$
R_{\mathrm{g}}:=E_{P}^{-1}\left(\frac{E}{E_{P}}\right)^{\frac{1}{D-3}}
$$

and where we have defined the fundamental Planck scale $E_{P}$ so that the reduced Compton wavelength agrees with the gravitational radius if the particle mass is equal to $E_{P}$. In this normalization, $D$-dimensional Einstein's gravitational constant $\kappa\left(G_{a b}=\kappa T_{a b}\right)$ is related to $E_{P}$ in the form

$$
\kappa=\frac{1}{2}(D-2) S_{D-2} E_{P}^{2-D},
$$

where $S_{n}=(n+1) V_{n+1}$ is the area of a unit $n$-sphere. Based on this picture, the total cross section of black hole formation is given by

$$
\sigma_{\mathrm{tot}}=\pi R_{\mathrm{g}}^{2}
$$

We consider the case in which these two particles pass through a transverse disk with a radius of $\nu R_{\mathrm{g}}$, where $\nu>1$. In this case, a black hole does not form. When these two 
particles pass through a disk of radius $\nu R_{\mathrm{g}}$ at almost the same time, they can be momentarily confined within a $(D-1)$-volume $V \simeq \pi \nu^{2} R_{\mathrm{g}}^{2} \times E^{-1} \times V_{D-4} E_{P}^{-(D-4)}$, where the second factor $E^{-1}$ is the uncertainty in the longitudinal position of each particle and $V_{D-4} E_{P}^{-(D-4)}$ is the volume in the extra dimensions. At this moment, the average energy density $\rho$ in this volume is estimated to be

$$
\rho \simeq \frac{E}{V}=\frac{E_{P}^{D}}{\pi \nu^{2} V_{D-4}}\left(\frac{E}{E_{P}}\right)^{2\left(\frac{D-4}{D-3}\right)} .
$$

If $\kappa \rho$ is larger than or equal to $\alpha E_{P}^{2}$, then, through the Einstein equation, the average Ricci tensor satisfies the criterion (11) for a border. The average density $\rho$ is a monotonically increasing function of the CM energy $E$. Thus, a sufficiently large CM energy can produce a visible border, since no black hole forms in this situation. The condition for realizing a border, $\kappa \rho \geq \alpha E_{P}^{2}$, and Eq. (17) lead to

$$
\nu^{2}<\nu_{\max }^{2}:=\frac{(D-2) S_{D-2}}{2 \pi \alpha V_{D-4}}\left(\frac{E}{E_{P}}\right)^{2\left(\frac{D-4}{D-3}\right)} .
$$

If the upper bound $\nu_{\max }^{2}$ is less than or equal to unity, the border will never be visible. Since $\nu_{\max }^{2}$ is an increasing function of the CM energy, if $E>\left[2 \pi \alpha(D-2)^{-1} S_{D-2}^{-1} V_{D-4}\right]^{\frac{1}{2}\left(\frac{D-3}{D-4}\right)} E_{P}$, then $\nu_{\max }^{2}$ is larger than unity, and the border will be visible.

TABLE I: Values of $\alpha \nu_{\max }^{2}$ when $E=14 \mathrm{TeV}$.

\begin{tabular}{cccccccc}
\hline \hline$E_{p}$ & $D=5$ & $D=6$ & $D=7$ & $D=8$ & $D=9$ & $D=10$ & $D=11$ \\
\hline $1 \mathrm{TeV}$ & 66.0 & 180 & 309 & 437 & 559 & 674 & 783 \\
\hline $14 \mathrm{TeV}$ & 4.71 & 5.33 & 5.89 & 6.40 & 6.87 & 7.31 & 7.73 \\
\hline \hline
\end{tabular}

The value of $\left(\nu_{\max }^{2}-1\right)$ expresses the ratio of the production rate of visible borders to that of black holes. The production rate of visible borders increases with increasing CM energy. In the case of $E=14 \mathrm{TeV}$ which is equal to the CM energy achieved by the LHC, if the fundamental Planck scale $E_{P}$ is equal to $1 \mathrm{TeV}$, the production rate of visible borders will be about $70 \alpha^{-1}$ times larger than the production rate of black holes for $D=5$, whereas it will about $800 \alpha^{-1}$ times larger than that of black holes for $D=11$ (see Table II). Although there is an ambiguous factor $\alpha$ in the criterion (11) for the border, the present result, which is based on dimensional analysis, implies that if black holes form at the LHC, more visible borders will be produced than black holes. 
$E$ will be larger than $E_{P}$ for the $\mathrm{LHC}$ if $E_{P}$ is about $1 \mathrm{TeV}$. This implies that the minimum uncertainty $E^{-1}$ in the longitudinal position will be smaller than the fundamental Planck length. If any structures smaller than the fundamental Planck length $E_{P}^{-1}$ are smeared out due to quantum gravitational effects, the uncertainty in the longitudinal direction should be estimated as the fundamental Planck length $E_{P}^{-1}$. If we adopt this assumption, the average energy density will be given by $\rho \simeq E_{P}^{D}\left(E / E_{P}\right)^{\frac{D-5}{D-3}} / \pi \nu^{2} V_{D-4}$. The corresponding $\nu_{\max }^{2}$ in this estimate is corrected only by the factor $E_{P} / E$ compared with the estimate (8). Hence, for this case too, we may expect that visible borders will appear at the LHC for $E \gtrsim E_{P}$. If it is necessary to account for quantum gravitational effects like these, we should regard such situations as situations in which the appearance of borders of spacetime is possible, even when the criterion (11) is not satisfied.

It is important to consider whether the visible border is gravitationally strong in the classical sense. In the framework of classical gravity (i.e., general relativity or Newtonian gravity), the codimension of singularity is closely related to the strength of its gravity. In four-dimensional spacetime, the codimension of a point singularity is three, that of a line singularity is two, and that of a plane singularity is one. As is well known, a singularity of codimension three is fatal as an object of finite size will be crushed by its tidal force [21, 22] if it has a non-zero gravitational mass. In the framework of general relativity, such singularities are enclosed by horizons. The tidal force of a codimension-two singularity is also strong enough to crush an object of finite size (except for conical singularities). However, since the gravity of a codimension-two singularity is generally weaker than that of a codimensionthree singularity, it can be naked if it is sufficiently long and almost straight [23, 24]. A codimension-one singularity is gravitationally weak and thus is not fatal. Since visible borders produced by high-energy collisions are compressed in two or more directions (the longitudinal and bulk directions), they will be gravitationally strong in the classical sense. Thus, nonlinear quantum gravitational effects are expected to occur at such visible borders.

For nongravitational interactions, the length scale of the physics that particle collisions reveal decreases with increasing CM energy. However, this relation may not hold when gravity plays an important role. If collisions on a super-Planckian energy scale necessarily produce only black holes (or in other words, super-Planckian phenomena necessarily appear through only black holes), higher CM energies will lead to long-range interactions rather than short-range interactions in the super-Planckian regime, because short-range interactions are 
hidden behind black holes and smeared out [4]. Probably, many researchers accept this picture. However, as we have seen in this paper, the larger CM energy of the particles may cause the formation of a visible border (i.e., a region with a super-Planckian average energy density that is not hidden behind the event horizon). A larger average density corresponds to a shorter curvature radius through the Einstein equations. Thus, super-Planckian physics may lead to an extremely short-range interaction through visible borders. At present, there is no precise prediction for what happens at the visible border since the theory of quantum gravity is not well developed. However, if the gravity in the vicinity of the visible border is sufficiently strong and is well described by the classical theory of gravity, semiclassical particles will be created in curved spacetime. If the democratic nature of gravity still prevails there, all kinds of particles will be emitted from them through a similar mechanism as the emission of Hawking radiation from a black hole. As mentioned above, if the visible border does not have any cutoff scales, the spectrum of emitted particles may be scale free, in contrast to the nearly black body radiation generated by Hawking evaporation of a black hole [25-27].

\section{Acknowledgements}

$\mathrm{KN}$ is grateful to $\mathrm{H}$. Ishihara and his colleagues in the astrophysics and gravity group at Osaka City University for helpful discussions and criticism. KN and TH thank T. Tanaka for very useful comments. TH and UM thank H. Tanaka for helpful comments. This work was partially supported by a Grant-in-Aid for Scientific Research from the Ministry of Education, Culture, Sports, Science and Technology, Japan [Young Scientists (B) 21740190 (TH) and $22740176(\mathrm{UM})]$.

[1] R. M. Wald, General Relativity (The University of Chicago Press, Chicago, 1984).

[2] N. Arkani-Hamed, S. Dimopoulos and G. Dvali, Phys. Lett. B 429, 263 (1998); I. Antoniadis, N. Arkani-Hamed, S. Dimopoulos and G. Dvali, Phys. Lett. B 436, 257 (1998).

[3] L. Randall and R. Sundrum, Phys. Rev. Lett. 83, 3370 (1999); Phys. Rev. Lett. 83, 4690 (1999).

[4] S. B. Giddings and S. Thomas, Phys. Rev. D 65, 056010 (2002).

[5] S. Dimopoulos and G. Landsberg, Phys. Rev. Lett. 87, 161602 (2001). 
[6] D. Ida, K. Oda and S. C. Park, Phys. Rev. D 67, 064025 (2003); Erratum-ibid.D 69, 049901 (2004); ibid. D 71, 124039 (2005); ibid. D 73, 124022 (2006).

[7] R. Penrose, Riv. Nuovo Cim. I, 252 (1969); reprinted in Gen. Relat. Grav. 34, 1141 (2002).

[8] P. S. Joshi, Gravitational Collapse and Spacetime Singularities (Cambridge Universe Press, England 2007).

[9] P. Brady and J. Smith, Phys. Rev. Lett. 75, 1256 (1995).

[10] L. M. Burko, Phys. Rev. Lett. 79, 4958 (1997).

[11] M. Dafermos, gr-qc/0401121.

[12] A. Ori, Phys. Rev. Lett. 68, 2117 (1992); ibid., 83, 5423 (1999).

[13] R. M. Wald, gr-qc/9710068.

[14] T. Harada and K. Nakao, Phys. Rev. D 70, 041501 (2004).

The definition of the border here is a bit different from the original one. However, this is simpler and clearer in the present context.

[15] S. A. Hayward, Class. Quantum Grav. 17, 1749 (2000).

[16] T. Piran, Phys. Rev. Lett. 41, 1085 (1978).

[17] T. Apostolatos and K. S. Thorne, Phys. Rev. D 46, 2435 (1992).

[18] K. Nakao and Y. Morisawa, Prog. Theor. Phys. 113, 73 (2005).

[19] K. Nakao, D. Ida and Y. Kurita, Phys. Rev. D 77, 044021 (2008).

[20] P. Kanti, Lecture Notes in Physics, 769, 387 (Springer, Berlin/Heidelberg 2009).

[21] F. J. Tipler, Phys. Lett. A 64, 8 (1977).

[22] A. Królak, J. Math. Phys. 28, 138 (1987).

[23] K. S. Thorne, in Magic Without Magic, ed. J. R. Klauder (Freeman, San Francisco, USA, 1972), p 231.

[24] T. Nakamura, S. L. Shapiro and S. A. Teukolsky, Phys. Rev. D 38, 2972 (1988).

[25] S. Barve, T. P. Singh, C. Vaz and L. Witten, Nucl. Phys. B 532, 361 (1998).

[26] T. Harada, H. Iguchi and K. Nakao, Phys. Rev. D 61, 101502 (2000); ibid., 62, 084037 (2000); Prog. Theor. Phys. 107, 449 (2002).

[27] T. Harada, H. Iguchi and K. Nakao, T. P. Singh, T. Tanaka and C. Vaz, Phys. Rev. D 64, 041501 (2001). 